\title{
Holographic (Quantum) Ways of Seeing, Hearing, Smelling, Memorizing and How to Use them for Diagnoses
}

\author{
Dr. Maria Kuman* \\ Holistic Research Institute, USA \\ *Corresponding author: Maria Kuman PhD, Holistic Research Institute, 1414 Barcelona, Knoxville, TN 37923, USA; Email: holisticare1@gmail.com
}

Submission: 海 April 16, 2018; Published: 海 June 01, 2018

\begin{abstract}
The article reveals that while our conscious is a digital computer, at subconscious level operates a quantum computer with a very high speed of computation and huge memory storage. It uses the coherent laser-type of waves of the human NEMF and works on the holographic principle, which allows very compact storage of everything we have seen, heard, smelled, or emotionally experienced during our lifespan. It also allows a very high speed of computation. The article discusses the possibility to use the quantum computer for diagnosis.
\end{abstract}

Keywords: Holographic images; Holographic memory; Quantum computers; Quantum diagnosis; Human NEMF; Subconscious

\section{Introduction}

In this article, we would show that we see not only with our eyes and we memorize not only with our conscious brain. For security reasons, we were ingenuously created with two types of memorizing and two types of vision. Even at quite severe damage of the brain surface, the past memories remain intact [1], [2,3]. This is because a full record of everything we have seen, heard, and smelled during our lifetime is also stored in deep areas of the brain, where our Subconscious is (for protection).

We know that the Subconscious contains a full record of our life from hypnosis, which puts the conscious to sleep and allows contact with the Subconscious [4]. Our sleep is non-stop alternative switches between light sleep, when the conscious is active (and dreams take place) and deep sleep when the subconscious is active. It seems that during these switches the visual, hearing, and etc. information recorded during the day in the conscious (on the surface of the brain) is scanned and sent for long-term storage in the deeper areas of the brain called the Subconscious.

\section{Our two types of memory}

Since we don't have conscious awareness of the function of our internal organs, the functioning of our organs is subordinated to the Subconscious, i.e. the Subconscious rules and regulates their function. It is deliberately done so because in crucial moments of our life, when our life is threatened and we are mobilized to survive, we don't want to be bothered with information about our internal organs.
All our organs are holographically represented on the surface of the skin: on our palms, feet, ears, and the irises of our eyes. The acupuncturists know that they can treat with acupuncture the points of the sick organ on any of these representations with equal success. Therefore, we have holographic representations of the organs on the surface of the body to tell us that our organs are ruled and regulated on holographic principle.

The famous mathematician von Neumann estimated that the human memory for a lifetime is about $2.8 .10^{20}$ bits of information [5,6]. What kind of memory should our subconscious have to be able to handle such large amount of information? Holographic memory (memory with coherent waves) allows the most compact record. Only with holographic type of memory can we explain the possibility for full lifetime record, as well as our ability to immediately recognize once known faces.

Thus, while our material brain memorizes on one principle, our subconscious memorizes through coherent waves on the holographic principle. Since our nonlinear electromagnetic field (NEMF) is wave abundant [7], it must be the one handling our lifetime holographic memory at subconscious level. Since our organs are subordinated to the subconscious, probably the NEMF rules and regulates their function as well from the subconscious.

By all means, it seems that this weak NEMF, which I spent 40 years of my life measuring, and which rules and regulates the functioning of our organs, is not the field produced by their functioning. Russian scientist's claim [8] that only when such 
NEMF enters a fertilized cell, the cell can be considered successfully fertilized and the embryo development of heart, lungs, brain, etc. can start. Thus, the NEMF is the one that creates the organs, and later rules, regulates, and synchronizes their function. It also rules (controls) the growing of our skin, nails, and hair and everything else in the body.

Dr. Keith Floyd, psychologist at Virginia Intermont College said: "If reality is nothing but a holographic illusion, the physical brain does not produce the mind. Rather, it is the mind that creates the appearance of the brain, as well as the body..." [6]. Indeed, the nonlinear electromagnetic field (NEMF) (of which the mind is part) creates the whole body, including the brain.

Through its waves, this NEMF records (memorizes) everything we see, hear, smell, or emotionally experience as holograms and store this information in the Subconscious. Since nonlinear electromagnetic field means also field that does not dissipate and field that can pass through walls or screens, at the end of life when the person dies, this non-dissipating NEMF leaves the body [6], but it carries the entire memory of the whole lifetime as a threedimensional holographic record.

\section{Our two types of vision}

Can we really see through waves? According to Hindu and Yoga texts, we can also see with our mind, not only with our eyes $[8,9]$. Russian scientists investigated V. Bronnikov, who could see with his mind [6]. First, when he sees with his eyes, the equipment registers impulse from his eyes to his brain. Second, when he is blindfolded and the equipment does not register impulse from his eyes to his brain, he could still see.

He could even see the object when it is behind a dense screen [6], which proves that nonlinear waves are involved because only nonlinear waves can pass through a screen. He could even see the object from different angles [6], which indicates that the vision is three-dimensional or holographic. Obviously, the seeing with the mind involves nonlinear coherent (laser type) waves and since our NEMF is rich of such waves, it must be involved in it.

Could our NEMF really see? In Bulgaria, there was a woman, Vanga, who was physically blind, but she could see with her mind. She would walk along the pathway in the garden and she would suddenly stop and pointing to a particular plant say to her niece: "You think that this is just a flower, but it is also an herb good for this and this disease." She was clairvoyant seeing with her mind inside the people's body, seeing their health problems, as well as their past and future. She was phenomenal and she was for years investigated by the Bulgarian Medical Academy.

I have spoken with many people who have been in a state of clinical death and came back. They all describe seeing their lifeless body lying on the bed, while hovering over it for three days. This means that during clinical death, when we say that the Spirit leaves, our NEMF leaves the body. Russian scientists did measure the NEMF and they found that it takes three days and three nights for this field to separate from the nervous system and leave [8]. However, if our
NEMF could see the physical body, this means that our NEMF has holographic vision, which is done through its coherent waves.

Even our hearing and smell are holographic. The German scientist Herman von Helmholtz 100 years ago showed that our ears detect waves and analyze frequencies [6]. Probably, the ears provide wave information of all we have heard, which is holographically memorized. Later, it was found that our ability to smell is also based on frequencies, so-called osmic frequencies [6]. Probably, our nose provides wave information of all we have smelled, which is holographically memorized.

\section{Who are the people that can see with their minds?}

Valerie [10] measured the frequencies of the NEMF of different people and found that it can vary rather dramatically. She found that clairvoyants' NEMF have frequencies between $400 \mathrm{~Hz}$ and $800 \mathrm{~Hz}$, people that can in trance connect with other dimensions have frequencies between $800 \mathrm{~Hz}$ and $900 \mathrm{~Hz}$, but some people can have NEMF with frequencies as high as $200,000 \mathrm{~Hz}$ and more. (However, for the human NEMF both the frequencies and the intensity of the field are important.) The higher the frequency of NEMF, the more spiritual the person is, and the better he sees with his mind $[7,8,10]$. This is an additional proof that the mind is an attribute of the human NEMF.

\section{Why does our movements and vision have wave nature?}

Considering the fact that waves run along the acupuncture meridians of our body, which was theoretically predicted by the author in 1983 [11] and experimentally proved by the Hungarian scientist in 1984 [12], all our movements should have wave nature. Indeed, the Russian scientist Bernstein found that the physical movements (dancing, walking, etc.) could be coded as waves and analyzed by the Furrier Method [6]. In 1979 the neurophysiologists of Berkley, Russell \& Karen De Valois [13], transformed the images of white and black cells into waveforms using the Furrier's Method [13]. They proved that the human brain responds to wave patterns.

\section{Where does the Pribram hypothesis for holographic brain stay?}

If the brain reacts to waves, where does the Pribram's hypothesis that the brain must work on the holographic principle stays? The fact that patients with surgically removed parts of their brain didn't suffer loss of memory drew his attention and he started researching where the memory is located. Karl Pribram found that even when he removed most of the cats' brain surface, the cats could still find their ways around. This means that their memories and ability of move remained intact, even when most of the brain surface was not there [1].

Pribram in collaboration with Karl Leshli also did experiments with rats [2]. Even when so much of the rat's brain surface was removed that they barely walked, their memories remained intact they could still find the shortest way in the labyrinth. Based on this, Pribram concluded that the memory is not local, but distributed in the whole brain. He always believed that the brain surface, and only the brain surface (the conscious) records or memorizes. 
Pribram's opponent, Pitsh, did experiments with salamanders [3]. He would cut the brain into pieces, mixed them, removed some, and the salamander would still retain its memory and recover completely. He gave up and accepted the holographic idea of Pribram. However, Pitsh experiments were another indication that the brain surface (the conscious) was not the one that created the holographic memory, but the NEMF at subconscious level, because even when the brain was so severely mistreated, the holographic memory was still there.

Also, people, who have been in a state of clinical death, when coming back still remember their relatives and friends, regardless that their brains were not functioning for three days [6]. The fact that their memories were not erased throws a shade over the widely accepted hypothesis that our brain surface (the conscious) creates the holographic memories, while de facto the subconscious and its NEMF create and store the holographic memories.

\section{The fast response belongs to our NEMF}

Toward the end of the last century, the San Francisco neurologists Benjamin Libet \& Bertram Feinstein [14] measured the time of their patients' response to a touch. Signal was registered in the brain 0.0001 second after the touch, but the patients were not consciously aware of this. This could only be a fast-propagating wave message related to our NEMF, but not related to our slow nervous system and brain surface. The button was pressed 0.1 second after the touch and this is the speed with which our nervous system operates.

Valerie Hunt [10] measured in California the speed of response with EEG (measuring the electrical activity on the surface of the head) and EMG (electromyography measuring the electrical activity of our muscles on the surface of the skin). She observed that EMG registers the fast response. It was done without conscious awareness, which meant it was done at subconscious level. Probably, it is done through the waves propagating on the surface of the skin, which were predicted by the author in 1983 [11] and experimentally found by Hungarian scientists in 1984 [12]. They are part of our NEMF.

Comparing EMG measurements, which characterize the human NEMF, with the EEG measurements, which characterize the activity of the brain surface, Valerie Hunt [10] saw that the human NEMF is much weaker, but reacts much faster than the nervous system, and she concluded that we overestimate the role of the conscious brain in our connection to the environment. She thinks that the brain is a good computer, be we cannot ascribe to it our creativity, imagination, spirituality, etc. "The Mind is not in the brain. It is in the energy field." [6] (Hunt's EMG measurements proved that NEMF rules the fast response and it operates at subconscious level).

The fast response, in our research [7] is related to the highspeed waves running on the surface of the body, which are part of our nonlinear electromagnetic field (NEMF). The lack of conscious awareness that this NEMF exist is a proof that it must be part of our subconscious. Since the NEMF is nonlinear coherent (laser type) waves, obviously our subconscious memory operates on the holographic principle. The compactness of this holographic memory is the only way to preserve the memory of a lifetime $(\sim$ 2.8.10 ${ }^{20}$ bits of information) [6] in the subconscious.

\section{Seeing with the mind for diagnosis}

Thus, the higher are the frequencies of NEMF of a person, the higher are his abilities to see with his mind. This is extremely important if the person is a medical doctor because the holographic seeing of his NEMF would allow him to see inside the body of the patient [15]. He wills not only see what is wrong with the patient, he would see what caused it, and he would see how to fix it.

I met at a conference in Bermuda Dr. Vitulkas from Greece, who was seeing with his mind. He was coming to the US to teach courses in a Naturopathic School and his students told me that his diagnoses were right more than $95 \%$ of the cases. The doctor told me how shocked one of his patients in Greece was when he told her that she is having heart troubles because of the problems she is having with her husband. Doctors usually don't say things like this, which explained her surprise, but she confirmed that she was indeed having troubles with her husband.

Shafica Karagula, neuropsychiatrist in Los Angeles, got interested in the 1960s of doctors doing nontraditional diagnostic with intuitive vision. After a few trials to contact such gifted doctor, finally she signed in as a patient. When in his office, she asked what he sees being wrong with her. He scanned her with his eyes and said she would need a surgery. She knew this because she already diagnosed herself and was just looking for confirmation [6]. He was right in every detail.

\section{Conclusion}

Thus, we can see with our eyes and the information is recorded in our conscious mind, which is our digital computer. But we can also see with our Mind, and this is done through our NEMF, which operate with waves at Subconscious level. Our Subconscious is our quantum computer, which operates with waves at thousand time's higher speed and memorizes on the holographic principle.

We are already on the way to create quantum computers and if so we should be ready to acknowledge the fact that our Subconscious operates as a quantum computer with holographic images. If in the creation of the quantum computers, we would have as a prototype the quantum computer in our Subconscious, we would have quantum computers with three-dimensional images capable to see what is inside the body, i.e. to diagnose with precision as the seeing with the mind doctors do.

\section{References}

1. Pribram K (1969) Brain and behavior. Penguin Books, UK.

2. Pribram K (1991) Brain and perception. Hillsdale, USA.

3. Pribram K (1994) Origins: brain and self-organization. Hillsdale, USA.

4. Weiss B (1988) Many lives, many masters, touchstone.

5. George Dyson Von Neumann's Universe. 
6. Tihoplav V, Tihoplav T (2007) Soznanie vselenoi. The Conscious of the Universe, Krilov Publisher, Russia.

7. Kuman M (2017) International Journal of Integrated Medicine 42(2): $97-112$.

8. Kuman M (2017) Same fields and dynamics in stars and man. Health and Happiness Books.

9. Kuman M (2015) Quantum mind and quantum growth. Health and Happiness Books.

10. Hunt V, www.biofieldenergy.org.
11. Kuman M (1983) Nonlinear mathematical model of one acupuncture meridian. VIII World Congress of Acupuncture, Sofia, Bulgaria.

12. Eory A (1984) Waves propagating along the acupuncture meridians detected. Acupuncture and Electro-Therapeutic Research 9: 217-223.

13. De Valois R, De Valois K (1979) Response to cortex cells to checkerboard patterns. J Physiol 291: 483-505.

14. Libet B, Feinstein B (1979) Timing for a conscious sensor experience. Brain 194: 191-222.

15. Kuman M (2005) Science speaks to god. Health and Happiness Books.
Creative Commons Attribution 4.0 International License

For possible submissions Click Here

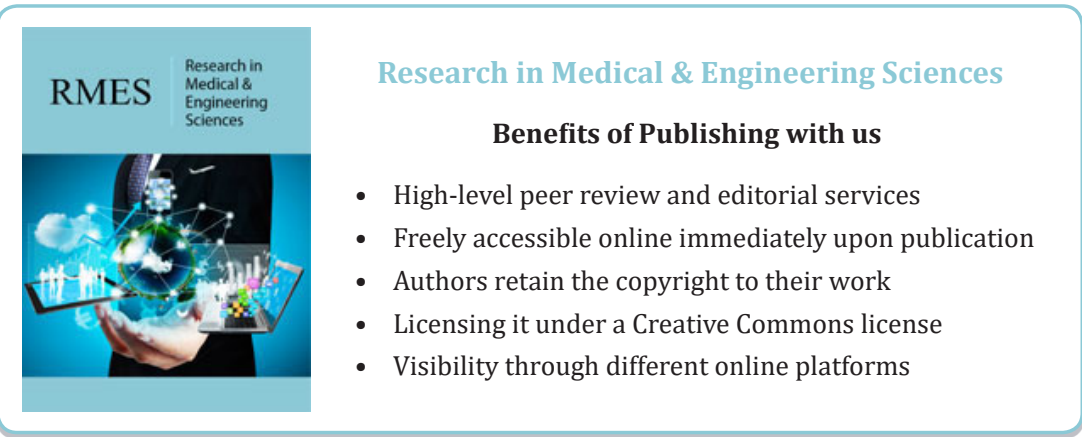

